\title{
Productivity change of Portuguese municipalities after local reforms
}

\author{
Juan Aparicio, Jose M. Cordero \& Jesus T. Pastor
}

To cite this article: Juan Aparicio, Jose M. Cordero \& Jesus T. Pastor (2017) Productivity change of Portuguese municipalities after local reforms, Applied Economics Letters, 24:12, 878-881, DOI: 10.1080/13504851.2016.1237738

To link to this article: http://dx.doi.org/10.1080/13504851.2016.1237738

册 Published online: 20 Oct 2016.

6 Submit your article to this journal $\pi$

凹 Article views: 25

Q View related articles $\asymp$

View Crossmark data $₫$ 


\title{
Productivity change of Portuguese municipalities after local reforms
}

\author{
Juan Aparicio ${ }^{a}$, Jose M. Cordero ${ }^{\mathrm{b}}$ and Jesus T. Pastor ${ }^{\mathrm{a}}$ \\ ${ }^{a}$ Center of Operations Research (CIO), University Miguel Hernandez of Elche (UMH), Alicante, Spain; ${ }^{b}$ Department of Economics, University of \\ Extremadura (UEX), Badajoz, Spain
}

ABSTRACT

This article examines the impact of the reforms introduced in the structure of the Portuguese local administration after the bailout agreement in 2011 on the efficiency and productivity of municipalities. In order to determine a measure of productivity change, we apply the global Malmquist productivity index to assess the performance of the 278 Portuguese mainland municipalities for the recent 6-year period 2009-2014. The overall results reveal that there was a decrease in the global productivity until 2013 followed by a notable increase in 2014 basically due to technological changes, thus it seems that the reforms have had a positive effect on the productivity of municipalities.

\section{KEYWORDS}

Efficiency; municipalities; productivity; local reforms;

Malmquist productivity

index

JEL CLASSFICATION $\mathrm{H} 71 ; \mathrm{H} 83 ; \mathrm{D} 24$

\section{Introduction}

This article focuses on the measurement of local government efficiency in Portugal. This country is an interesting case study because municipalities are all subject to the same political and administrative rules and laws and have the same policy instruments and resources at their disposal. Moreover, as a consequence of the political compromise taken by the Portuguese government in the context of the economic adjustment programme established in 2011 after the European Union/International Monetary Fund (EU/IMF) bailout process, multiple reforms have been implemented in the structure of municipalities with the aim of reducing costs and improving efficiency. The key commitments were to reduce grants to municipalities, decrease the local debt limit and cut back staffing levels in municipalities (Teles 2016). Another important matter was the reorganization of the administrative map by reducing the number of civil parishes ${ }^{1}$ (freguesias) in accordance with the terms of the Law 11-A/2013.

The aim of this research is to assess the efficiency and productivity of Portuguese local governments during this transformation process. To do this, we apply the global Malmquist productivity index (MPI) developed by Pastor and Lovell (2005), which allows determining productivity change over time while several interesting properties are satisfied. In particular, and in contrast to the traditional Malmquist index $\left(M^{G}\right)$, the global Malmquist is always feasible, even under Variable Returns to Scale, and meets circularity. The global Malmquist can also be decomposed into the usual measures of efficiency change and technical change as well as scale efficiency. This article contributes to the existing literature about the global performance assessment of municipalities which is mainly represented by studies referred to a single year (see Cruz and Marques 2014 for a recent literature review).

\section{Methodology}

Let us consider a panel of $j=1, \ldots, n$ municipalities and different time periods $(k=1, \ldots, K)$. In this framework, we need to define two technologies to establish the relationship between inputs $x \in \Re_{+}^{m}$ and outputs $y \in \Re_{+}^{s}$. On the one hand, the contemporaneous benchmark technology is defined as $T^{k}=\left\{\left(x^{k}, y^{k}\right): x^{k}\right.$ can produce $\left.y^{k}\right\}$. On the other hand, the global benchmark technology is defined as $T^{G}=\operatorname{conv}\left\{T^{1} \cup T^{2} \cup \ldots \cup T^{K}\right\}$. Moreover, we will use a subscript linked to the technology for

CONTACT Jose M. Cordero jmcordero@unex.es

${ }^{1}$ A civil parish is a subdivision of a municipality with its own elected bodies, possibly a neighbourhood or city district, a group of hamlets, a village, a town or an entire city, which has limited powers.

(c) 2016 Informa UK Limited, trading as Taylor \& Francis Group 
denoting which type of returns to scale is being assumed: $T_{c}^{k}$ for Constant Returns to Scale (CRS) and $T_{v}^{k}$ for Variable Returns to Scale. In this way, we have $T_{c}^{G}=\operatorname{conv}\left\{T_{c}^{1} \cup T_{c}^{2} \cup \ldots \cup T_{c}^{K}\right\} \quad$ and $T_{v}^{G}=\operatorname{conv}\left\{T_{v}^{1} \cup T_{v}^{2} \cup \ldots \cup T_{v}^{K}\right\}$. Additionally, the Shephard input distance function calculated on the production possibility set $T$ is defined as $D_{i}^{T}(x, y)=\sup \{\lambda:(x / \lambda, y) \in T\}$.

Following Pastor and Lovell (2005), the global Malmquist input-based productivity index is defined as

$$
M^{G}\left(x^{t}, y^{t}, x^{t+1}, y^{t+1}\right)=\frac{D_{i}^{T_{c}^{G}}\left(x^{t}, y^{t}\right)}{D_{i}^{T_{c}^{G}}\left(x^{t+1}, y^{t+1}\right)}
$$

The global MPI was originally decomposed into two sources of productivity change: efficiency change (EC) and technical change (TC). EC represents the changes in the relative technical efficiency levels of municipalities, that is, their ability to catch up with the benchmarks capturing the municipal technology spread, whereas TC measures the shift of the frontiers suggesting technology changes over the examined period. In this article, we decompose this index considering also a third component: scale efficiency change (SC). To do that, we follow the approach proposed by Ray and Desli (1997) in order to break down the contemporaneous Malmquist index into three terms. Therefore, we decompose $M^{G}$ as follows:

$$
\begin{gathered}
M^{G}\left(x^{t}, y^{t}, x^{t+1}, y^{t+1}\right)=\frac{D_{i}^{T_{v}^{t}}\left(x^{t}, y^{t}\right)}{D_{i}^{T_{v}^{t+1}}\left(x^{t+1}, y^{t+1}\right)} \\
\underbrace{\left[\frac{D_{i}^{T_{v}^{t+1}}\left(x^{t+1}, y^{t+1}\right)}{D_{i}^{T_{v}^{G}}\left(x^{t+1}, y^{t+1}\right)} \cdot \frac{D_{i}^{T_{v}^{G}}\left(x^{t}, y^{t}\right)}{\left.D_{i}^{T_{v}^{t}}\left(x^{t}, y^{t}\right)\right]}\right.}_{E C} . \\
\underbrace{\frac{D_{i}^{T_{c}^{G}}\left(x^{t}, y^{t}\right) / D_{i}^{T_{v}^{G}}\left(x^{t}, y^{t}\right)}{D_{i}^{T_{c}^{G}}\left(x^{t+1}, y^{t+1}\right) / D_{i}^{T_{v}^{G}}\left(x^{t+1}, y^{t+1}\right)}}_{T C}
\end{gathered}
$$

$E C$ is the usual efficiency change indicator, comparing two contemporaneous Shephard's distance functions, while TC captures the change experienced in the best practice gaps between $T_{v}^{G}, T_{v}^{t+1}$ and $T_{v}^{t}$. Finally, $S C$ is the ratio of scale efficiencies of the bundles, $\left(x^{t}, y^{t}\right)$ and $\left(x^{t+1}, y^{t+1}\right)$, with respect to the global technology. Additionally, and as usual, $M^{G}\left(x^{t}, y^{t}, x^{t+1}, y^{t+1}\right)>1$ indicates an improvement in total productivity, $M^{G}\left(x^{t}, y^{t}, x^{t+1}, y^{t+1}\right)<1$ denotes a decline and $M^{G}\left(x^{t}, y^{t}, x^{t+1}, y^{t+1}\right)=1$ implies an unchanged productivity growth. The same interpretation can be applied to EC, TC and SC.

The global Malmquist index satisfies a set of properties that make it interesting. First, like any fixed base index, $M^{G}$ is circular. Second, the index and its components provide a single measure with no need to take the geometric mean of disparate adjacent period measures. Third, the TC and scale terms of $M^{G}$ are immune to the usual linear programming infeasibilities associated with the traditional contemporaneous Malmquist index, which frequently generates a problem of indetermination in practice.

\section{Data and variables}

In our empirical analysis, we assess the relative efficiency of the 278 municipalities located in mainland Portugal over the period 2009-2014. The island municipalities were omitted because they have some specific financial and fiscal benefits that could cause comparability problems with other mainland local governments. ${ }^{2}$ The studied period covers the whole term of local governments elected in 2009 as well as the first year of the new governments elected in $2013 .{ }^{3}$

The output indicators selected to estimate the efficiency levels of Portuguese municipalities represent the set of services that the municipalities provide to the population. In particular, we use the quantity of water supplied, the urban waste collected and the building permits issued as well as the total resident population as a proxy for the rest of administrative tasks (De Borger and Kerstens 1996). The variables used as inputs represent the resources consumed by local governments in the provision of the above services. Specifically, we separate personnel expenditures from all other spending, including operational and capital (nonfinancial investment and capital grants) expenditures. All these data

\footnotetext{
${ }^{2}$ Afonso and Fernandes (2008) provide similar arguments to support their decision to exclude the island municipalities from their analysis of Portuguese local governments.

${ }^{3}$ The Portuguese local election of 2013 took place on 29 September, thus the new governments started to operate at the end of the year.
} 
were gathered from municipality annual reports and the National Statistics Institute (INE in its Portuguese acronym).

\section{Empirical results}

Table 1 presents the average values of the global Malmquist index and its three components, while Fig. 1 illustrates their evolution over the examined period. We can observe that there was an increase in productivity in average terms after the first election year (2009); then the productivity levels stagnated and subsequently declined in the period 2012-2013. Finally, there was another great improvement in the period after the local elections (2013-2014). This evidence is in line with recent results focused on efficiency measurement of Portuguese local governments (Cordero et al. 2016). If we closely examine the decomposition of these changes in productivity, we can notice that the first increase was mainly driven by EC, while the second growth experienced in the last period was mostly prompted by TC. Therefore, it seems that the reforms introduced in the structure of local governments in 2013, mainly represented by the reduction of the number of civil parishes, have had a positive impact on the productivity of municipalities in average terms. Actually, the technical efficiency also increased after the first restrictive measures were adopted in 2011, but subsequently there was a significant decline between 2012 and 2013.

Table 1. Average values of the global MPI and its components (2009-2014).

\begin{tabular}{lccccc}
\hline & $2009-2010$ & $2010-2011$ & $2011-2012$ & $2012-2013$ & $2013-2014$ \\
\hline$M^{G}$ & 1.053716 & 0.991788 & 1.018627 & 0.924893 & 1.067234 \\
EC & 1.038310 & 1.029740 & 0.981285 & 0.994143 & 1.027628 \\
SC & 1.000625 & 0.990702 & 0.975304 & 0.976795 & 0.989657 \\
TC & 1.005581 & 0.968962 & 1.061231 & 0.953828 & 1.049431 \\
\hline
\end{tabular}

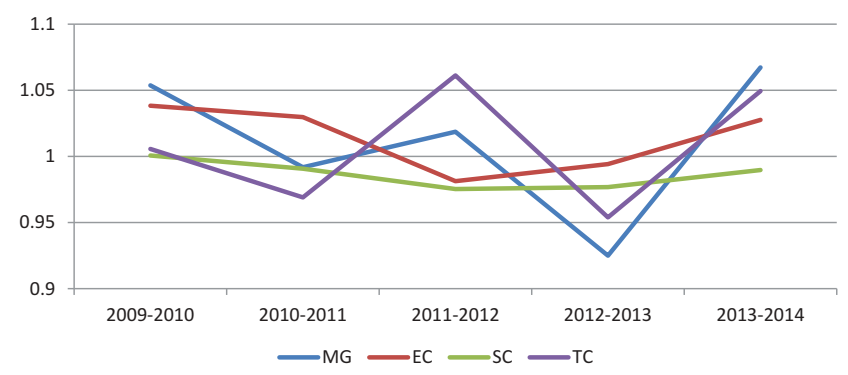

Figure 1. Evolution of the average values of the global MPI index and its components.
More interesting results can be derived by exploring the distribution of the efficiency scores across local governments of different sizes. Following Carvalho et al. (2014), we classify them into three categories using the number of inhabitants as a proxy: small (less than 20000 inhabitants), medium (from 20000 to 100000 inhabitants) and large (more than 100000 inhabitants). Figure 2 presents the evolution of the global Malmquist index and their components over the examined period for the three categories of municipalities and the whole sample. Figure 2(a) reveals that the evolution of the global productivity index over the years is similar for all types of municipalities, although the decrease and increase experienced in the last two periods are more pronounced for medium and small local governments. If we examine the components, it is possible to identify that the relative situations of large municipalities have worsened during these years in terms of EC (Fig. 2(b)). This is the reason that explains their lower global productivity in the last period despite the fact that these municipalities have higher levels of scale efficiency (Fig. 2(c)) and a greater improvement in TC in that period (Fig. 2(d)).

\section{Conclusions}

By using a global productivity Malmquist index, we examine the effect of the recent reforms introduced in the structure of the local governments in Portugal. The overall results reveal that there has been a significant improvement on productivity caused by a technology change after the implementation of those measures, thus it seems that they have had a positive effect. Likewise, we find that this improvement has been more substantial in small- and medium-sized municipalities. Large municipalities suffered a decline in their technical efficiency levels over the evaluated period, thus their improvement in global terms has been less significant.

\section{Acknowledgements}

Juan Aparicio and Jesus T. Pastor would like to express their gratitude to the Spanish Ministry for Economy and Competitiveness for supporting this research through grant MTM2013-43903-P, and Jose M. Cordero also acknowledges the support from the same institution through grant ECO201453702-P. 
a. Global Malmquist index
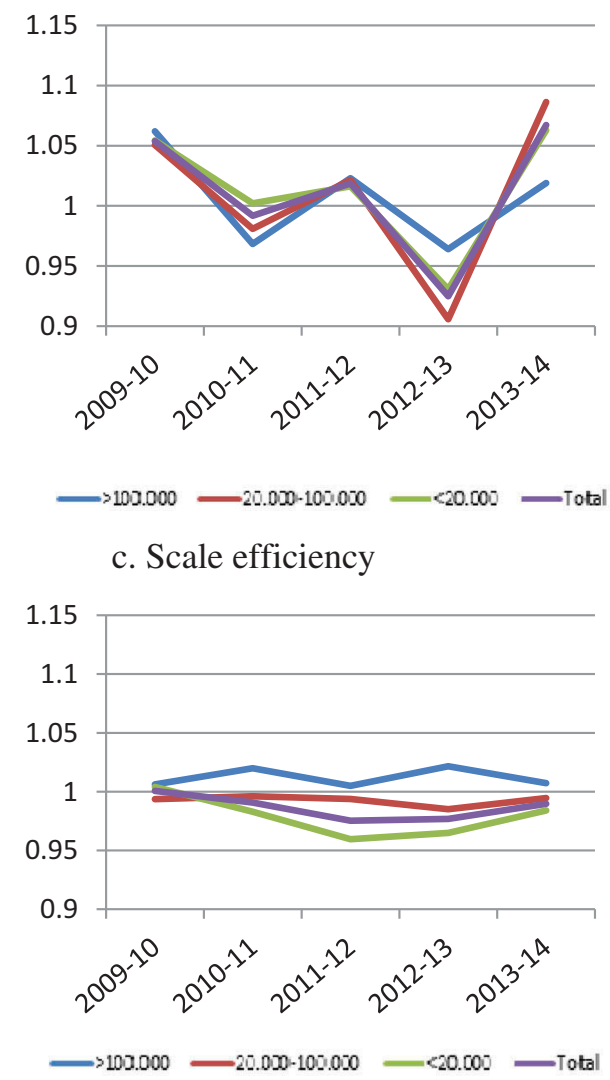

b. Efficiency change

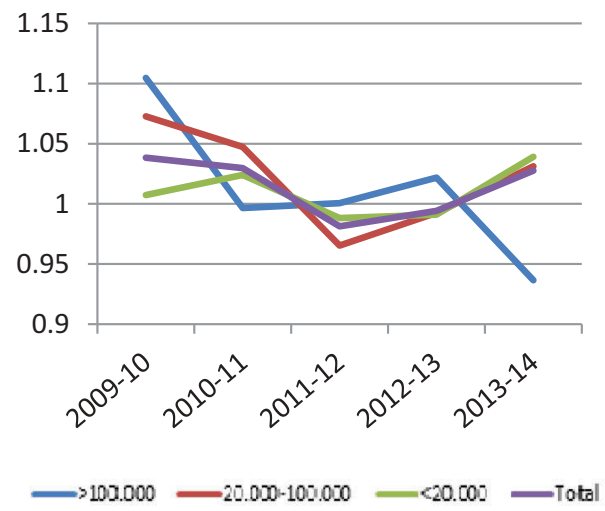

d. Technical change

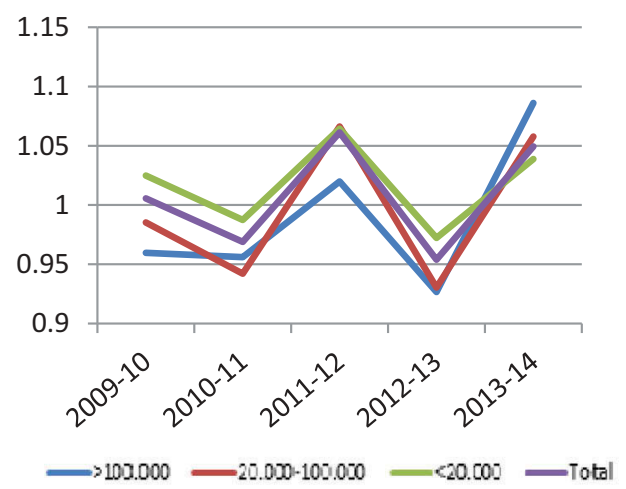

Figure 2. Differences in global productivity and its components according to the size: (a) global Malmquist index; (b) efficiency change; (c) scale efficiency; (d) technical change.

\section{Disclosure statement}

No potential conflict of interest was reported by the authors.

\section{Funding}

This work was supported by the Ministerio de Economía y Competitividad under grants MTM2013-43903-P and ECO2014-53702-P.

\section{References}

Afonso, A., and S. Fernandes. 2008. "Assessing and Explaining the Relative Efficiency of Local Government." The Journal of Socio-Economics 37 (5): 1946-1979. doi:10.1016/j.socec.2007.03.007.

Carvalho, J., F. Mj, P. Camões, and S. Jorge. 2014. Anuário financeiro dos municípios portugueses 2013 (Financial Yearbook of Portuguese Municipalities 2013). Lisbon: Câmara dos Técnicos Oficiais de Contas (CTOC).
Cordero, J. M., F. Pedraja-Chaparro, E. C. Pisaflores, and C. Polo. 2016. Efficiency Assessment of Portuguese Municipalities Using a Conditional Nonparametric Approach, MPRA paper $\mathrm{n}^{\circ}$ 70674. https://mpra.ub.uni-muenchen.de/70674/

Cruz, N. F., and R. C. Marques. 2014. "Revisiting the Determinants of Local Government Performance." Omega 44: 91-103. doi:10.1016/j.omega.2013.09.002.

De Borger, B., and K. Kerstens. 1996. "Cost Efficiency of Belgian Local Governments: A Comparative Analysis of FDH, DEA, and Econometric Approaches." Regional Science and Urban Economics 26 (2): 145-170. doi:10.1016/0166-0462(95)02127-2.

Pastor, J. T., and C. A. K. Lovell. 2005. "A Global Malmquist Productivity Index.” Economics Letters 88 (2): 266-271. doi:10.1016/j.econlet.2005.02.013.

Ray, S. C., and E. Desli. 1997. "Productivity Growth, Technical Progress, and Efficiency Change in Industrialized Countries: Comment." The American Economic Review 87 (5): 1033-1039.

Teles, F. 2016. "Local Government and the Bailout: Reform Singularities in Portugal." European Urban and Regional Studies 23 (3): 455-467. doi:10.1177/0969776413517249. 